\title{
Experiencia de gamificación en Docencia Universitaria: aprendizaje activo y entretenido
}

Eva Serna $^{\mathrm{a}}$, María Dolores Mauriciob, Teresa San Miguel ${ }^{\mathrm{a}}$ y Javier Megías ${ }^{\mathrm{a}}$.

aDepartamento de Patología, Universitat de València. Eva.serna@uv.es, Teresa.Miguel@uv.es,

Javier.Megias@uv.es bepartamento de Fisiología, Universitat de València.

m.dolores.mauricio@uv.es

\begin{abstract}
The main objective of this paper is to describe the experience developed in university teaching using gamification as a teaching tool. Gamification is the term used to refer to the application of game mechanics in areas in which gaming is not typically used.

Our working hypothesis is based on the idea that gaming can be a new methodology that favors the settlement and internalizing the concepts taught by making the learning process and integrate asset and related contents of an attractive and motivating way for students.In order to assess the experience of students we have spent an anonymous survey and we have analyzed their answers. Our conclusion is that gamification has a good welcome and it is able to increase the level of attention and knowledge consolidation.
\end{abstract}

Keywords: gamification, active learning, motivation, university teaching, educational innovation, educational intervention

\begin{abstract}
Resumen
El objetivo fundamental del presente trabajo es describir la experiencia desarrollada en docencia universitaria usando la gamificación como herramienta docente. El término de gamificación se utiliza para referirnos a la aplicación de mecánicas de juego en ámbitos que normalmente no son lúdicos.

Nuestra hipótesis de trabajo se basa en que el juego puede ser una nueva metodología que favorezca el asentamiento e interiorización de los conceptos impartidos haciendo que el proceso de aprendizaje sea activo y que integren y relacionen contenidos de un modo atractivo y motivante para el alumnado. Con el fin de valorar la experiencia del alumnado distribuimos una encuesta anónima y analizamos sus respuestas. Nuestra conclusión es que la gamificación tiene una buena acogida y consigue aumentar el grado de atención y de consolidación de conocimientos.
\end{abstract}

Palabras clave: gamificación, aprendizaje activo, motivación, docencia universitaria, innovación educacional, intervención educacional 


\section{Introducción}

El aprendizaje mediante el juego en la docencia universitaria no es un método clásicamente utilizado, sin embargo podría proporcionar buenos resultados tal y como hemos demostrado en estudios previos (Mauricio, 2015). La ludificación o gamificación son términos utilizados para referirnos a la aplicación de mecánicas de juego en ámbitos que normalmente no son lúdicos (Deterding, 2011; Llagostera, 2012 y Werbach, 2014).

La gamificación en la educación es un enfoque serio para motivar la acción, promover el aprendizaje y resolver problemas (Kapp, 2012) , pero hay pocas evidencias empíricas de la eficacia de gamificación en la educación. Existen estudios que han mostrado su eficacia en términos de involucrar a los estudiantes en las actividades no curriculares (Fitz - Walter, 2012 ) y la promoción de cambios de comportamiento con el fin de aumentar la participación del alumnado. La gamificación podría conseguir aumentar la motivación y el esfuerzo, y hacer más partícipes a los alumnos en el proceso de aprendizaje. Según Cortizo y colaboradores (Cortizo, 2011) al realizar actividades basadas en juegos se pueden impulsar cambios de hábito tanto en los estudiantes como en los profesores, especialmente, este cambio puede hacer que el estudiante pase a tener un papel más activo en el proceso de enseñanza-aprendizaje y no un simple receptor de conocimiento, algo que persigue el Espacio Europeo de Educación Superior (EEES).

La adecuación al EEES ha supuesto una profunda renovación no sólo en la estructura curricular, sino también en la metodología de enseñanza. El EEES aboga por cambiar el modelo, que se supere el enfoque de la transmisión de conocimientos, y se apueste por un planteamiento en el que el profesor se convierta en facilitador del aprendizaje y el alumno en constructor de su propio proceso formativo, con la finalidad de que aprenda a aprender y se favorezca su autonomía. Para afrontar este nuevo reto, la motivación por aprender se hace más necesaria que nunca (Romero y Pérez Ferra, 2009), ya que es una de las variables que más incidencia tiene en el bajo rendimiento del alumnado, a juicio de los profesores, y en opinión de los propios alumnos, quienes llaman la atención, precisamente, sobre la falta de estrategias de motivación por parte del profesorado (Tejedor y García-Valcárcel, 2007). Por tanto, resulta evidente la necesidad de que estos últimos adopten nuevas formas de pensar, de sentir y de actuar favorecedoras de la motivación de los estudiantes (Martínez González, 2011). En este sentido, nuestras experiencias previas con la gamificación han demostrado un aumento de la motivación intrínseca de los estudiantes por la asignatura y un aumento en el número de aprobados. Además en anteriores cursos hemos puesto en marcha distintas modalidades de juego y la que más éxito suele tener entre nuestros estudiantes es la de "minijuegos". Se trata de un lote juegos variados (puzzles, relación de conceptos, etc) de corta duración que han de resolverse en grupo y en el menor tiempo posible como si de una competición se tratase (Mauricio, 2015). Con esta práctica la clase se vuelve muy dinámica, divertida y se aclaran muchas dudas.

(cc) EY-NC-ND 2016, Universitat Politècnica de València 
A raíz de todo lo expuesto anteriormente, nuestra propuesta de trabajo se basa en la idea de que el juego puede ser una nueva metodología y una herramienta que favorezca el asentamiento e interiorización de los conceptos impartidos haciendo que el proceso de aprendizaje no sea meramente memorístico, sino que integren y relacionen contenidos de un modo atractivo y motivante.

\section{Objetivos}

El objetivo fundamental del presente trabajo es describir la experiencia desarrollada usando la gamificación como herramienta docente. Para ello los subobjetivos son:

1- Aplicar una sesión de juego en un alumnado de Grado de Odontología

2- Valorar y comparar los puntos fuertes y débiles de la aplicación del juego desde el punto de vista del profesorado y del alumnado.

3- Analizar mediante una encuesta anónima si se han consolidado conceptos, si la atención ha sido mayor durante la sesión de gamificación comparada con la clase teórica- práctica, así como obtener información acerca de si el juego en grupo resulta más provechoso que el individual.

\section{Desarrollo de la innovación}

\section{Descripción de la experiencia de gamificación:}

La experiencia se llevó a cabo con el alumnado matriculado en la asignatura de Biología Celular, Grupo A, del Grado de Odontología de la Universitat de Valencia en el curso académico 2015-2016. El número total de alumnos matriculados fue de 87, de los cuales 74 participaron en la sesión de gamificación realizada.

El perfil del alumnado según datos del aula virtual fueron:

Tabla 1. Perfil del alumnado

\begin{tabular}{cc}
\hline Edad & Porcentaje (\%) \\
\hline 18 & 50.57 \\
19 & 22.99 \\
20 & 3.45 \\
21 & 3.45 \\
22 & 4.60 \\
23 & 2.30 \\
24 & 2.30 \\
25 & 1.15 \\
28 & 1.15
\end{tabular}

(cc) EY-Nc-ND 2016, Universitat Politècnica de València

Congreso In-Red (2016) 


\begin{tabular}{ll}
29 & 1.15 \\
31 & 1.15 \\
33 & 1.15 \\
34 & 1.15 \\
37 & 1.15 \\
46 & 1.15 \\
51 & 1.15 \\
\hline
\end{tabular}

Las clases prácticas para este Grado de Odontología consisten en 6 sesiones de 2 horas de duración cada una.

1. Manejo del microscopio óptico.

2. Técnicas de adaptación: Coloración. Citoquimia.

3. Tipos celulares.

4. Cultivos celulares.

5. División celular.

6. Gametogénesis.

En cada una de estas sesiones se proporciona al alumnado una base teórica para que $a$ posteriori el alumno pueda observar unas preparaciones e imágenes al microscopio de acuerdo con los objetivos de la asignatura.

Al finalizar las prácticas de la asignatura se hizo una sesión de repaso aplicando un minijuego. Esta sesión de repaso es importante porque posteriormente el alumnado realiza un examen práctico, con lo que le ayuda a consolidar sus conocimientos.

El mini-juego consistió en hacer equipos de 3-4 personas y a los que se les proporcionó 4 imágenes que se habían explicado en prácticas y que el alumnado previamente habia trabajado y visualizado al microscopio óptico.

En esta sesión de repaso, para tener una vision amplia de todas las prácticas, se eligió una imagen de cada una de las prácticas, en total 4. La práctica 1 "Manejo del microscopio óptico" no se incluye ya que ese día no se proporcionan preparaciones fundamentales cara a su evaluación. La última práctica de “Gametogénesis” tampoco fue incluida en el juego porque se había impartido ese mismo día.

Posteriormente, se proporcionó a cada equipo un listado de todas las prácticas realizadas como síntesis y para así poder elegir mediante la realización del mini-juego la preparación que correspondía a cada imágen proyectada (Figura 1).

(cc) EY-NC-ND 2016, Universitat Politècnica de València 
Práctica 2 - Técnicas de adaptación: Coloración. Citoquimia.

Preparación III .1

Preparación III .2

Preparación III .3

Preparación III .4

Práctica 3 - Tipos celulares

Preparación IV .1

Preparación IV .2

Preparación IV .3

Práctica 4 - Cultivos celulares

Preparación V .1

Preparación V .2

Preparación V .3

Preparación V .4

\section{Práctica 5 - División celular}

Preparación VIII .1

Preparación VIII .2

Preparación VIII .3

Preparación VIII .4

Práctica 6 - Gametogénesis

Preparación I .1

Preparación I .2

Preparación I .3
Tumor ascítico: giemsa

Tumor ascítico: P.A.S.

Tumor ascítico: Feulgen

Tumor ascítico: lactato deshidrogenasa
Sangre humana: giemsa

Sangre de anguila: giemsa

Frotis vaginal: Papanicolaou

Cultivo primario por explantes: giemsa

Cultivo secundario (fibroblastos): giemsa

Línea celular establecida sarc-2: giemsa

Línea celular establecida mel-1: giemsa

Raíz de cebolla: carmín acético

Raíz de cebolla: corte semifino, azul de toluidina

Cromosomas humanos: giemsa

Cromosomas de un tumor experimental: giemsa

Corte de ovario: hematoxilina-eosina

Corte de testículo: hematoxilina-eosina

Espermatozoides: Papanicolaou

Figura 1: Listado de las diferentes preparaciones visualizadas en las prácticas del curso.

(c)) EY-NC-ND 2016, Universitat Politècnica de València

Congreso In-Red (2016) 
Con el fin de comparar el trabajo individual con el grupal, se procedió a la realización de los juegos primero de forma individual y después en grupo. Es decir, la resolución de las dos primeras imágenes fue individual y la resolución de las otras dos imagenes restantes fue en grupo.

En el caso de la resolución individual se eligieron las prácticas 3 y 4 correpondientes a "Tipos Celulares” y “Cultivos Celulares”. Mientras que las prácticas 2 y 5 se utilizaron para resolverlas en grupo: “Coloración. Citoquimia” y “División cellular”.

Las imágenes fueron proyectadas a la vez para todo el alumnado y de una en una (Figura 2).
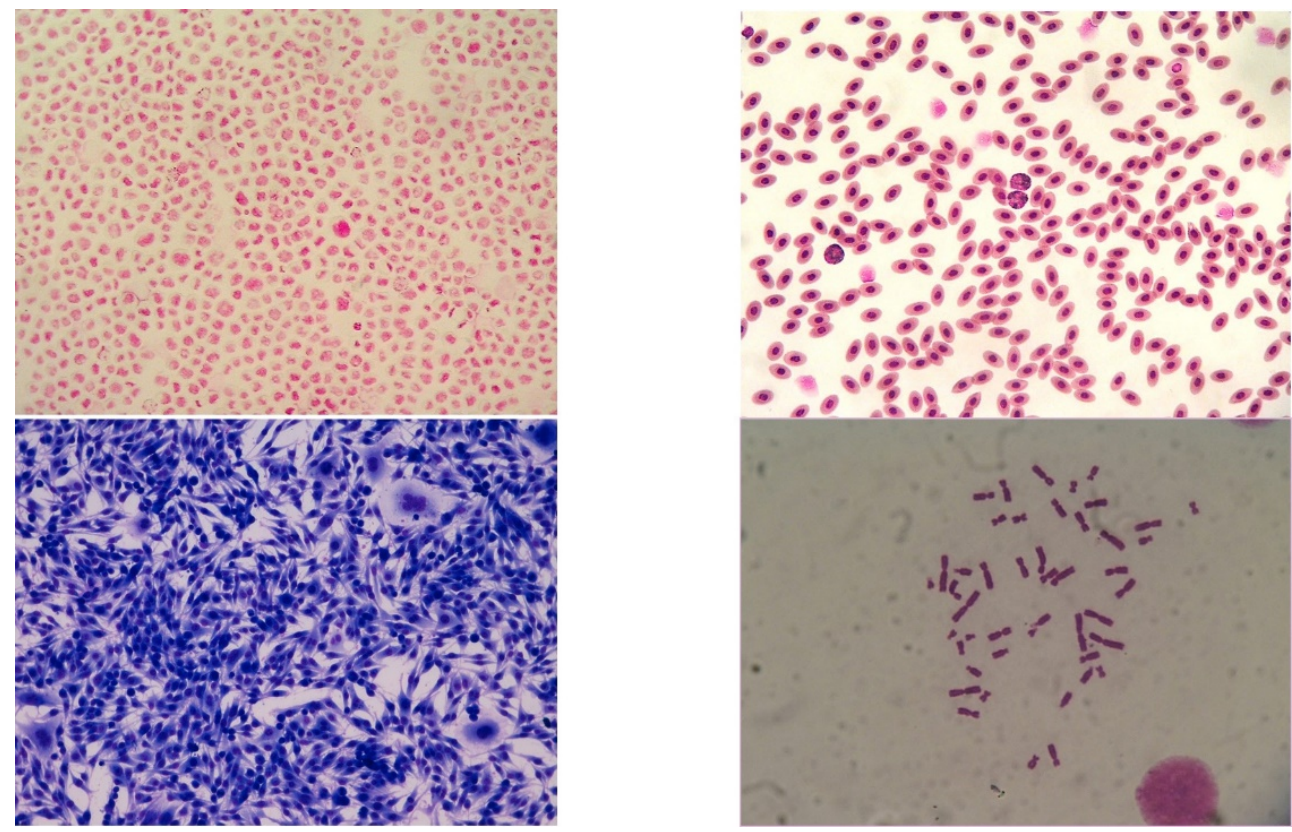

Figura 2: Imágenes de las preparaciones proyectadas durante la gamificación. Fotografias tomadas por el Grupo de Biología Celular del Departamento de Patología de la Universidad de Valencia.

La resolución también es imagen por imagen.

Cuando se resuelve la primera preparación o imagen, ya sea individual o grupal, se realiza una explicación a modo de resumen de cómo diferenciarlas con más imágenes no proyectadas pero que han sido trabajadas y visualizadas por el alumnado de esa misma práctica. Y se pasa a la segunda imagen, así hasta resolver las 4 imágenes.

La idea es que la resolución sea en el menor tiempo posible y que la persona o grupo que sabe su respuesta levante la mano para que el profesorado coordine la fase de respuesta y poder ser comentada. 
Finalmente, después de realizar la experiencia del juego y para evaluar de qué manera los alumnos percibieron esta sesión de gamificación, elaboramos una encuesta que respondieron de forma anónima. En ésta se formulaban las siguientes preguntas:

1.- ¿Consideras que el hecho de repasar las preparaciones mediante el juego te ha ayudado a entender mejor los conceptos?

a) Sí

b) No

c) No lo sé

2.- ¿Consideras que es más fácil mantener la atención durante una clase de juego que durante una clase teórica/práctica?

a) Sí

b) No

c) No lo sé

3.- El hecho de repasar la materia en grupo te resulta más provechoso que hacerlo de forma individual?

a) Sí

b) No

c) No lo sé

4.- Escribe 3 aportaciones positivas y negativas sobre el juego que hemos hecho.

\section{Resultados}

Según nuestra experiencia docente en el aula de prácticas la experiencia de la gamificación fue muy positiva. Los alumnos participaron activamente y se divirtieron. Fue una clase de repaso muy dinámica y diferente. La sesión de repaso no supuso más de 10 minutos de duración de toda la sesión total.

A continuación se detallan algunas apreciaciones que hemos ido recogiendo y que se desprenden de nuestra experiencia para que el juego se perfeccione en un futuro:

1- La apreciación del profesorado se sintetizaría en que la sesión de repaso fue muy corta y las contestaciones eran muy rápidas.

2- Para garantizar el trabajo en equipo cuando se realiza el juego grupal, había que dejar esperar a que todos los miembros del equipo pudieran aportar su contribución y dar una respuesta consensuada por todos sus miembros.

3- La cantidad de integrantes del equipo es buena para que todos participen y se impliquen en el juego.

Por otra parte, analizamos las respuestas de 74 encuestas, porque aunque fue una encuesta voluntaria y anónima, todos los estudiantes eligieron participar.

Los resultados fueron los siguientes (Figura 3):

(cc)) EY-NC-ND 2016, Universitat Politècnica de València

Congreso In-Red (2016) 

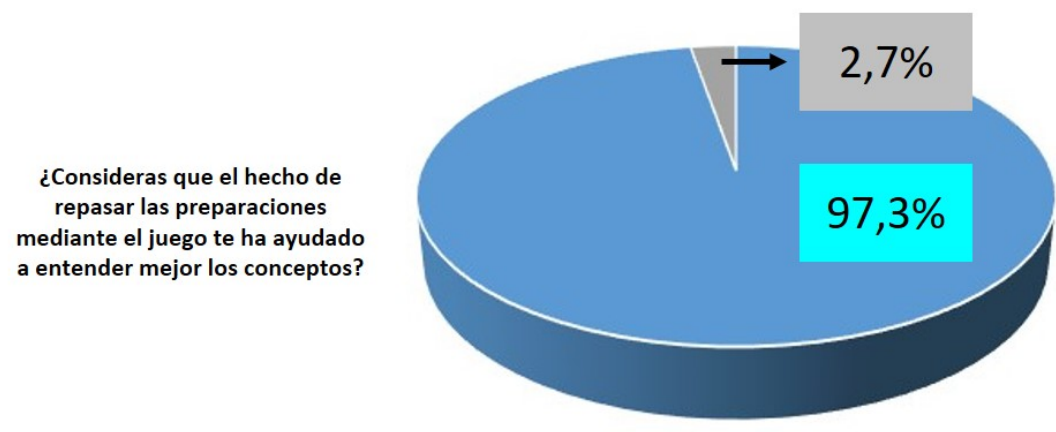

॥I $=$ NO $=$ NOLOSÉ
¿Consideras que es más fácil mantener la atención durante una clase de juego que durante una clase teórica/práctica?

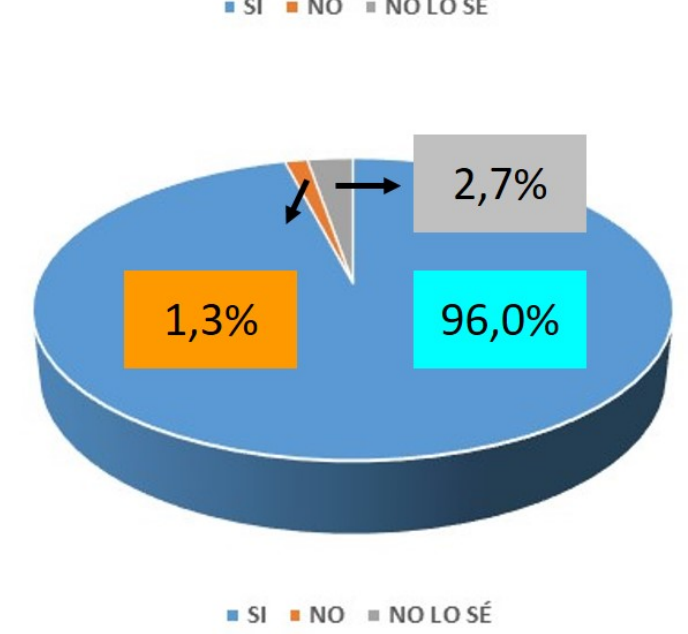

¿El hecho de repasar la materia en
grupo te resulta más provechoso
que hacerlo de forma individual?

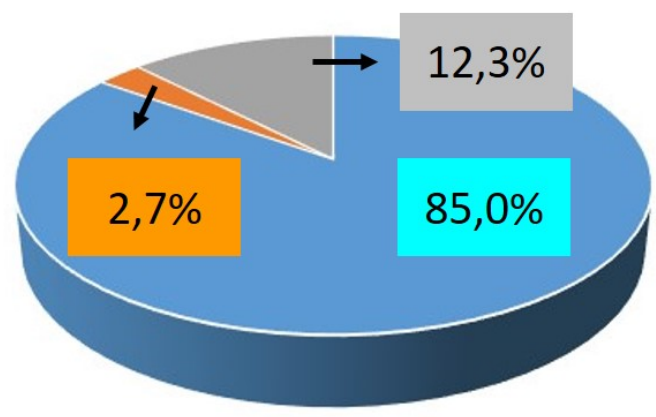

nSI $=$ NO $=$ NOLOSÉ

Figura 3: Respuestas del alumnado a las preguntas pasadas en la encuesta anónima.

A la primera pregunta:

1.- ¿Consideras que el hecho de repasar las preparaciones mediante el juego te ha ayudado a entender mejor los conceptos?

(cc) EY-Nc-No 2016, Universitat Politècnica de València 
72 respondieron que “Sí” y 2 “No lo sé”.

A la pregunta: 2 - $-\dot{¿}$ Consideras que es más fácil mantener la atención durante una clase de juego que durante una clase teórica/práctica?

71 respondieron que “Sí”, 1 que “No” y 2 "No lo sé”.

En contestación a la pregunta $3 .-¿$ El hecho de repasar la materia en grupo te resulta más provechoso que hacerlo de forma individual?

62 dijeron que "Sí”, 2 que “No”, 9 “No lo sé” y hubo una respuesta en blanco.

Por último la encuesta incluyó una pregunta más abierta con el fin de obtener información directa del alumnado y hacer así un análisis más completo de nuestra experiencia con la gamificación.

Los aspectos positivos y negativos a juicio de los alumnos sobre esta metodología se recogen en las tablas 2 y 3 respectivamente.

Tabla 2. Aspectos positivos de la gamificación

\begin{tabular}{cc}
\hline $\mathbf{N}^{\mathbf{o}}$ aportaciones & Pros \\
\hline 55 & Repasa la materia /autoevaluación \\
35 & Aprendizaje y afianzamiento de conceptos \\
16 & Es dinámico, entretenido \\
13 & Aclara y resuelve dudas \\
12 & Sirve para preparación para examen \\
10 & Fomenta el trabajo en equipo \\
8 & Sirve para identificar mejor preparaciones \\
7 & Es interactivo \\
6 & Es motivador \\
5 & Profundización de la materia \\
4 & Es didáctico/educativo \\
4 & Aumenta atención/concentración \\
3 & Es tranquilizador \\
3 & Es competitivo \\
2 & No es monótono \\
2 & aprendido (dibujos) \\
\hline
\end{tabular}

(cc) EY-Nc-ND 2016, Universitat Politècnica de València

Congreso In-Red (2016) 
Tabla 3. Aspectos negativos de la gamificación

\begin{tabular}{cc}
\hline $\mathbf{N}^{\mathbf{o}}$ aportaciones & Contras \\
\hline 36 & No hay nada en contra \\
18 & Breve/poco tiempo \\
18 & No se repasan todas las preparaciones \\
10 & No se hace a menudo \\
7 & El juego es muy rápido \\
5 & So hay posibilidad de repasar antes \\
3 & Se resuelve en voz alta \\
2 & Es demasiado distendido \\
2 & Hay miedo a expresarse y participar \\
1 & Posibles distracciones \\
1 & Qi el juego no interesa no se participa \\
1 & Quiere más trabajos en equipo \\
1 & \\
1 &
\end{tabular}

Los resultados de la encuesta indican más aportaciones positivas que negativas. También destacar que existen 36 aportaciones que indican que no encuentran nada negativo en este mini-juego.

Dentro de los aspectos positivos un porcentaje muy alto comentan que la gamificación les sirve para repasar y autoevaluarse (55 aportaciones), así como para aprender y afianzar conceptos (35 aportaciones).

Como aspectos negativos, a los alumnos les parece que la gamificación debería consumir más tiempo de la sesión total, que se repasaran todas las preparaciones y que se utilizara como herramienta de trabajo más a menudo.

(cc) EY-NC-ND 2016, Universitat Politècnica de València 


\section{Conclusiones}

En primer lugar, cabe destacar que todos los alumnos que han participado en las sesiones de gamificación lo han hecho voluntariamente, es decir, no se ha incentivado la participación en el juego con un valor añadido sobre la evaluación final. Esta metodología se ha puesto en práctica con la intención de mejorar la transferencia de conocimientos y la consolidación de los mismos.

Nuestra experiencia con gamificación con alumnos del Grado de Fisioterapia (Mauricio, 2015) nos desveló que los mini-juegos gustaban a los estudiantes porque se trabajaba con un grupo reducido, lo que favorecía la participación de todos los miembros, comparado con otras modalidades de juego como el "concurso", que implicaba separar la clase en dos equipos y habitualmente éstos resultaban muy numerosos, por lo que no se conseguía la implicación de todos sus integrantes. Para el profesorado, la modalidad de mini-juego es mucho más práctica ya que, aunque la clase sea numerosa, el profesor hace tantos lotes como equipos haya. Es por ello, que aplicamos una versión de dicha modalidad al alumnado del Grado de Odontologia para comparar si la herramienta se podía hacer extensiva a otros Grados.

La conclusión general es que la gamificación tiene una muy buena acogida en el alumnado del Grado de Odontología. Se considera que su uso ayuda a entender mejor los conceptos, a afianzarlos, proporciona más atención que en una clase teórica-práctica y, en general, al alumnado, les gusta más trabajar en equipo que de manera individual.

Desde el punto de vista del tiempo invertido en el aula, la sesión de mini-juegos se realizó en 10 minutos, algo a tener en cuenta, ya que normalmente no se dispone de horas extra para realizar actividades como ésta. Sin embargo, al alumnado le supo a poco y la aportación negativa más extendida que nos devuelven es que la gamificación fue corta y que se debería hacer más a menudo en el aula.

En la experiencia del profesorado, resultó una metodología sencilla aplicable sin ningún problema al aula y que se podría hacer más larga para que el alumnado pueda disfrutarla y repasar más conceptos y preparaciones. Se propone utilizar el doble de tiempo, es decir, 20 minutos de la sesión total.

Desde el punto de vista del alumnado, éste enfatiza que sirve como autoevaluación, aprendizaje y repaso de la materia; que era uno de nuestros principales objetivos. Pero, además, cabe destacar la cantidad de sustantivos positivos que nos devuelven: dinámico, entretenido, divertido, interactivo, motivador, didáctico, competitivo, tranquilizador, interesante, recreativo, provechoso, productivo,etc... Esto hace pensar que el clima creado en el aula fue el adecuado para aprender a aprender. Esta metodología activa provoca que el alumnado se vuelva participativo y responsable de su aprendizaje. El profesor adquiere el papel de motor-motivador que ayuda al alumno a reflexionar, razonar, crear sus respuestas.

Nuestra experiencia coincide con los planteamientos de Piñeiro-Otero y Costa-Sánchez (Piñeiro-Otero, 2015) donde indicen en la importancia de la gamificación en el ámbito educativo, y concretamente en la docencia universitaria, donde destacan que es una estupenda 
oportunidad para incentivar la motivación, la participación y la creación de un conocimiento compartido. Siendo aún muy escasos los trabajos existentes en la literatura.

Por todo ello, podemos concluir que la gamificación es una herramienta educativa eficaz en el ámbito universitario, y concretamente, en grados que abarcan el Área de la Salud. Con el juego se consigue aumentar el grado de atención e interiorizar y consolidar los conocimientos aprendidos.

\section{Referencias}

CORTIZO PÉREZ, JC. CARRERO GARCÍA, F. MONSALVE PIQUERAS, B. VELASCO COLLADO, A. DÍAZ DEL DEDO, LI. y PÉREZ MARTÍN, J. (2011). “Gamificación y Docencia: Lo que la Universidad tiene que aprender de los Videojuegos”. En Retos y oportunidades del desarrollo de los nuevos títulos en educación superior. VIII Jornadas Internacionales de Innovación Universitaria. Madrid. Disponible en <http://www.josek.net/publicaciones/JIU2011-Preprint.pdf>

DETERDING, S. KHALED, R. NACKE, LE. DIXON, D. (2011). “Gamification: Towards a Definition” en CHI 2011. Vancouver. AMC 978-1-4503-0268-5/11/05

FILZ-WALTER, Z.,. TJONDRONEGORO, D., WYETH, P. (2012) “A gamified mobile application for engaging new students at university orientation”. Proceedings of the 24th Australian ComputerHuman Interaction conference, ACM, Melbourne, Australia pp. 138-141

KAPP, K.M. (2012). The gamification of learning and instruction: Game-based methods and strategies for training and education. San Francisco: Pfeiffer.

LLAGOSTERA, E. (2012). “On Gamification and Persuasion” en XI SB Game. SBC-Proceeding of SB Games. Brasilia.Games for change-Full papers.12-21.

MARTÍNEZ GONZÁLEZ J.A. (2011). "La motivación para aprender en el espacio europeo de educación superior”. Cuadernos de Educación y Desarrollo, Vol. 3 (25). http://www.eumed.net/rev/ced/25/jamg.htm [Consulta: 09-03-2016].

MAURICIO, M.D, SERNA, E y VALLES, S.L. (2015) "Experiencias en la aplicación de la gamificación en $1^{\circ}$ Curso de Grado de Ciencias de la Salud” en Congreso de Innovación Educativa y Docencia en Red. Universitat Politècnica de València. Disponible en $<$ http://ocs.editorial.upv.es/index.php/INRED/>

PIÑEIRO-OTERO, T. Y COSTA-SÁNCHEZ, C. (2015). “ARG (juegos de realidad alternativa). Contribuciones, limitaciones y potencialidades para la docencia universitaria”. Comunicar, Vol. 44(22), pp.141-148.

ROMERO, M. Y PÉREZ FERRA, M. (2009). “Cómo motivar a aprender en la universidad: una estrategia fundamental contra el fracaso académico en los nuevos modelos Educativos”. Revista Iberoamericana de Educación, Vol. 51, pp. 87-105.

TEJEDOR, F.J. Y GARCÍA-VALCÁRCEL, A. (2007). “Causas del bajo rendimiento del estudiante universitario (en opinión de los profesores y alumnos). Propuestas de mejora en el marco del EEES”. Revista de Educación, Vol. 342, pp. 443-473

WERBACH, K. (2014). “(Re) Defining Gamification: A Process Approach” en Spagnolli, A. Persuasive Technology: Persuasive Technology lecture Notes in Computer Science Switzerland. Springer International Publishing. (8462) pp 266-272.

(cc) EY-NC-ND 2016, Universitat Politècnica de València 